\title{
Establishment and characterization of gastric carcinoma cell clones expressing LMP2A of Epstein-Barr virus
}

\author{
JUNG SEON SEO ${ }^{1}$, SE MO JUN ${ }^{1}$, SANG WOOK KWON ${ }^{1}$, \\ IL-HOAN OH${ }^{2}$, TAI-GYU KIM ${ }^{3}$ and SUK KYEONG LEE ${ }^{1}$ \\ ${ }^{1}$ Department of Biomedical Sciences, Research Institute of Immunobiology, \\ ${ }^{2}$ Department of Cellular Medicine, Catholic Cell Therapy Center, ${ }^{3}$ Department of Microbiology, \\ College of Medicine, The Catholic University of Korea, Seoul 137-701, Republic of Korea
}

Received August 11, 2009; Accepted September 23, 2009

DOI: 10.3892/ijmm_00000307

\begin{abstract}
Although Epstein-Barr virus (EBV) has been detected in $5-15 \%$ of gastric carcinoma (GC) cases, the mechanism by which EBV contributes to its tumorigenesis remains unclear. Only a subset of EBV latent proteins such as EBV nuclear antigen-1 and latent membrane protein $2 \mathrm{~A}$ (LMP2A) are expressed in EBV-associated GC (EBVaGC) cases. In this study, to elucidate the role of LMP2A in the tumorigenesis of EBVaGC, we established permanent cell lines expressing LMP2A. The LMP2A gene was cloned from a naturally EBV-infected EBVaGC cell line, SNU-719 and transduced into an EBV-negative GC cell line, AGS, using a retroviral vector. The sequence of SNU-719 LMP2A showed several conserved variations compared to that of the prototype EBV strain B95-8 LMP2A. Four of seven established cell clones expressed LMP2A protein at detectable levels. These cell clones did not show enhanced cell growth compared to control cells in normal or low serum-containing medium. Furthermore, LMP2A expression had no effect on colony forming ability of the cell clones in soft agar. Our results suggest that LMP2A alone has little effect on tumorigenesis of EBVaGC.
\end{abstract}

\section{Introduction}

Epstein-Barr virus (EBV) infection is associated with several human malignancies of lymphoid and epithelial origin, including Burkitt's lymphoma (BL), Hodgkin's lymphoma (HD), nasopharyngeal carcinomas (NPC) and gastric

Correspondence to: Dr Suk Kyeong Lee, Department of Biomedical Sciences, Research Institute of Immunobiology, Catholic University of Korea, 505 Banpo-dong, Seocho-gu, Seoul 137-701, Republic of Korea

E-mail: sukklee@catholic.ac.kr

Key words: Epstein-Barr virus, gastric carcinoma, latent membrane protein $2 \mathrm{~A}$, tumorigenesis, retroviral vector carcinoma (GC) (1-3). Latent EBV infection can cause tumor initiation and propagation through the expression of viral genes that affect the cell cycle, apoptosis and immune response. EBV expresses several viral proteins with transforming ability including EBV nuclear antigen (EBNA)2, EBNA3A, EBNA3C and latent membrane protein 1 (LMP1).

EBV-positive gastric carcinoma (EBVaGC) is the most prevalent among EBV-associated malignancies and EBV association is found in 5-15\% of GC worldwide (4-6). As EBV is found in almost all tumor cells of EBVaGC and the EBV genome is present as a monoclonal episomal form $(5,7), \mathrm{EBV}$ may act as an oncovirus to cause gastric carcinogenesis. However, it is still unclear whether the presence of EBV is the cause or the result of gastric carcinogenesis because none of the known viral oncogenes are expressed in EBVaGC $(8,9)$.

EBVaGC exhibits a modulated latency I EBV infection, in that the expression of viral latent genes is restricted to EBNA-1, Epstein-Barr encoded RNAs (EBERs) and transcripts from the BamHI-A region (BARTs). In addition, LMP2A is frequently expressed in EBVaGC but LMP1 and LMP2B are not $(5,10)$. LMP2A is a transmembrane protein that inhibits normal B-cell signal transduction by mimicking an activated B-cell receptor (BCR) (11-13). LMP2A activates the phosphatidylinositol 3-kinase (PI3K)/Akt pathway, which normally provides a survival signal in response to BCR signaling $(14,15)$. Previous studies reported that LMP2A contributes to maintenance of viral latency but it is not required for in vitro infection or growth transformation of primary B cells $(16,17)$. However, it has been argued that LMP2A is essential for survival and continued proliferation of $\mathrm{B}$ cells, even though LMP2A is dispensable for B cell transformation if the cells express BCR (18). LMP2A may also be involved in EBVpositive gastric carcinogenesis; by enhancing transformation and epithelial cell motility as well as inhibiting epithelial cell differentiation (19-21). Conversely, LMP2A did not induce anchorage-independent cell growth in the human epithelial cell line (HaCaT) (22). LMP2A expression in the epidermis of transgenic mice had no effects on the growth and differentiation of epithelial cells (23). Therefore, there are inconsistent issues about the oncogenic role of LMP2A in EBV-associated epithelial tumors. 
Previously, we reported that SNU-719 is a gastric carcinoma cell line that is naturally infected with EBV and that this cell line expresses EBNA-1, LMP2A and EBERs but not LMP1 similar to most EBVaGC cases (24). To examine the oncogenic potential of LMP2A in gastric epithelial cells, the LMP2A gene from SNU-719 was cloned and transduced into an EBV-negative gastric cancer cell line, AGS, using a retroviral vector and the growth ability of cell clones expressing LMP2A was analyzed.

\section{Materials and methods}

Cell culture. SNU-719 is a naturally derived EBV-infected GC cell line (24,25). AGS is an EBV-negative GC cell line (26) that was used to transduce the LMP2A gene from SNU-719 using a retroviral vector. SNU-719 and AGS cell lines were obtained from Korean Cell Line Bank (Seoul, Korea). Cells were cultured in RPMI-1640 medium (Gibco BRL, Grand Island, NY, USA) supplemented with $10 \%$ fetal bovine serum (FBS), penicillin and streptomycin (100 units/ml (Invitrogen, Carlsbad, CA, USA), and fungizone ( $250 \mu \mathrm{g} / \mathrm{ml}$, Gibco BRL). The AGS-EBV cell line, which is AGS infected with a recombinant Akata virus (27), was maintained in RPMI-1640 medium supplemented with $10 \%$ FBS and $400 \mu \mathrm{g} / \mathrm{ml} \mathrm{G418}$ (Gibco BRL).

Cloning of LMP2A from SNU-719 in a retroviral vector. Coding sequence of SNU-719 LMP2A was amplified by reverse transcription-polymerase chain reaction (RT-PCR). Briefly, RNA was extracted from the SNU-719 cells using RNAzol B (Tel-Test, Friendswood, TX, USA) and RT was conducted in $20 \mu \mathrm{l}$ reaction volumes at $37^{\circ} \mathrm{C}$ for $50 \mathrm{~min}$. For PCR, $5 \mu 1$ of RT product was added to $50 \mu 1$ of PCR mixture containing $10 \mathrm{mM}$ Tris- $\mathrm{HCl}$ (pH 9.0), $10 \mathrm{mM} \mathrm{KCl}, 15 \mathrm{mM}$ $\mathrm{MgCl}_{2}, 8 \mathrm{mM}\left(\mathrm{NH}_{4}\right)_{2} \mathrm{SO}_{4}, 1 \%$ NP-40, $1 \mathrm{mM}$ dNTPs, 1 unit of pfu DNA polymerase (Invitrogen) and 25 pmol of PCR primers specific for LMP2A (5'-AGAATTCATGGGGTCCCTAGA AATG-3' and 5'-ATACTCGAGCTTTATACAGTGTT GCG-3'). The PCR mixtures were subjected to 30 cycles of PCR in a thermocycler (Perkin Elmer, Foster City, CA, USA). Each cycle consisted of a denaturing step at $94^{\circ} \mathrm{C}$ for $30 \mathrm{sec}$, an annealing step at $55^{\circ} \mathrm{C}$ for $60 \mathrm{sec}$ and an extension step at $72^{\circ} \mathrm{C}$ for $120 \mathrm{sec}$. The amplified DNA products were separated by electrophoresis in a $1 \%$ agarose gel with $0.5 \mathrm{X}$ Tris-acetate-EDTA (TAE) buffer and stained with $0.5 \mu \mathrm{g} / \mathrm{ml}$ ethidium bromide. Bands of interest were excised from the gel, purified using a gel extraction kit (Qiagen, Valencia, CA, USA) and ligated into pGEM easy T-vector (Promega, Madison, WI, USA). The LMP2A gene was then subcloned into a retroviral vector, $\mathrm{pMIG}$, which contains enhanced green fluorescence protein (EGFP) as a reporter (28). The constructed plasmids were sequenced by the dideoxynucleotide chain termination method using an $\mathrm{ABI}$ prism $^{\mathrm{TM}}$ Terminator Cycle Sequencing Ready Reaction Kit and an ABI Prism 3770 Genetic analyzer (Perkin Elmer).

Virus infection. To produce retrovirus encoding LMP2A, $293 \mathrm{~T}$ cells $\left(1 \times 10^{6} / \mathrm{ml}\right)$ were seeded in a $60-\mathrm{mm}$-diameter dish and cultured for $24 \mathrm{~h}$ prior to transfection. A LMP2A gene expression construct or the empty pMIG vector was then transfected into the cells by a calcium phosphate method (29).
After $24 \mathrm{~h}$, the medium was removed and fresh medium added. The conditioned medium was harvested following an additional $72 \mathrm{~h}$ of incubation and filtered through a $0.45-\mu \mathrm{m}$-pore size membrane to collect virus solution. AGS cells $\left(0.5 \times 10^{5} /\right.$ well $)$ were seeded in a 6 -well plate $24 \mathrm{~h}$ before infection. The cells were then incubated with $4 \mathrm{mg} / \mathrm{ml}$ of polybrene and $1 \mathrm{ml}$ of virus solution for $24 \mathrm{~h}$. The medium was then changed and GFP expression was evaluated.

Isolation of EBV-infected cell clones. AGS cells expressing the LMP2A gene were sorted using fluorescence-activated cell sorting (FACS) based on the expression of the GFP marker. GFP-expressing cells were then serially diluted and seeded in 96-well plates. A microscope was used to determine the wells containing only a single cell. These wells were then marked and maintained at $37^{\circ} \mathrm{C}$ until the cells grew to $\sim 70 \%$ confluence, at which time they were transferred to 12-well plates and expanded for further experiments.

Detection of LMP2A expression by RT-PCR. The expression of LMP2A gene in the established cell clones was examined by RT-PCR using specific primers for LMP2A (5'-TACTGA GTAGAGTTGTGTAT-3' and 5'-CATGTTAGGCAAATTG CAAA-3', $280 \mathrm{bp}$ ). The RT-PCR product was separated by electrophoresis in a $2 \%$ agarose gel. The integrity of the RNA and RT-PCR reaction was evaluated by a parallel amplification of glyceraldehydes 3-phosphatedehydrogenase (GAPDH).

Detection of LMP2A protein by Western blot analysis. To detect LMP2A protein, cell lysate in RIPA buffer (50-100 $\mu \mathrm{g})$ was mixed with $2 \mathrm{X}$ sodium dodecyl sulfate (SDS) sample buffer and heated at $70^{\circ} \mathrm{C}$ for $5 \mathrm{~min}$. Next, the samples were subjected to $10 \%$ SDS-polyacrylamide gel electrophoresis and the separated proteins were transferred to a polyvinylidene fluoride membrane (Millipore, Billerica, MA, USA). Rat polyclonal antibody against LMP2A (15F9, GSF-Forschungszentrum fur Umwelt und Gesundheit, Munchen, Germany) was used as a primary antibody. After washing, the blots were incubated with horseradish peroxidase (HRP)-conjugated anti-rat (Amersham Biosciences, Piscatawy, NJ, USA) secondary antibody for $2 \mathrm{~h}$ at room temperature. Protein bands were visualized using an enhanced chemiluminescence detection system (Amersham Bioscience). A 1:1000 dilution of anti- $\alpha$-tubulin antibody (Calbiochem, San Diego, CA, USA) was used to confirm comparable loading.

Colony formation assay on soft agar. A single cell suspension in $3 \mathrm{ml}$ of $0.3 \%$ SeaKem LE agarose (Cambrex Bio Science Rockland, Inc., ME, USA) was over-laid onto $3 \mathrm{ml}$ of $0.6 \%$ base agarose in 6 -well plates $\left(1.5 \times 10^{4} /\right.$ well). To prevent drying out and to supply nutrients, $0.5 \mathrm{ml}$ of complete medium was added to the top of the agarose layers once a week. After 3 weeks of incubation at $37^{\circ} \mathrm{C}$, the resulting colonies were stained with $0.005 \%$ crystal violet for $30 \mathrm{~min}$ at room temperature and then washed with de-ionized water. The colonies were observed under an inverted microscope and counted using the UTHSCSA Image Tool 3.0 program (http://ddsdx.uthscsa.edu). The colony-forming efficiency was calculated by dividing the number of colonies with the number of plated cells. 
A

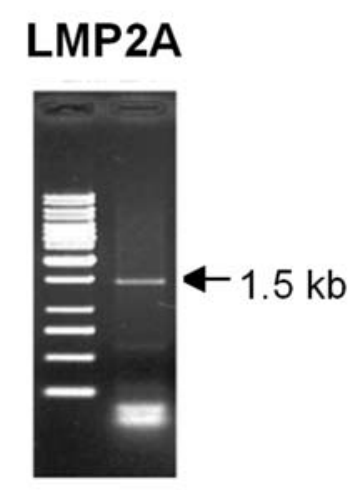

B

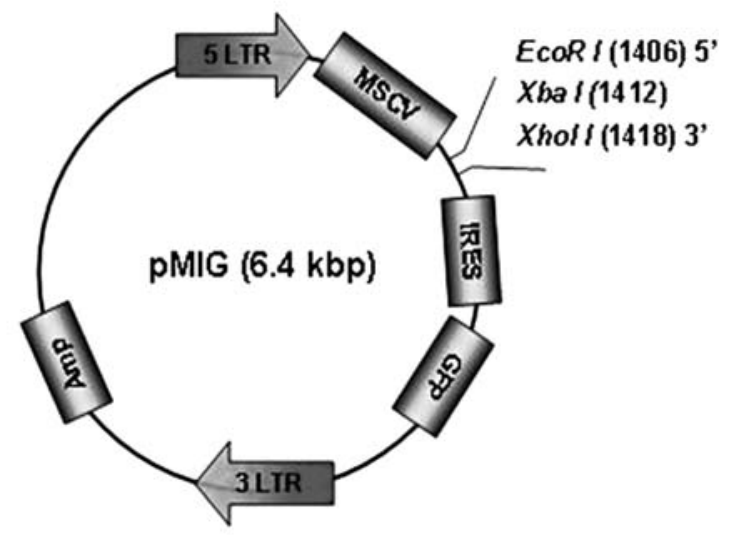

Figure 1. Cloning of LMP2A gene from SNU-719 in a retroviral vector. Total RNA was extracted from SNU-719 cells. The cDNA of LMP2A was amplified by RT-PCR. The expected size of the PCR product is marked on the right (A). Schematic diagram of the retroviral vector, pMIG. The pMIG has an internal ribosomal entry site (IRES) cassette and downstream enhanced GFP cDNA which can be used to select transduced cells by FACS (B).

\section{LMP2A}

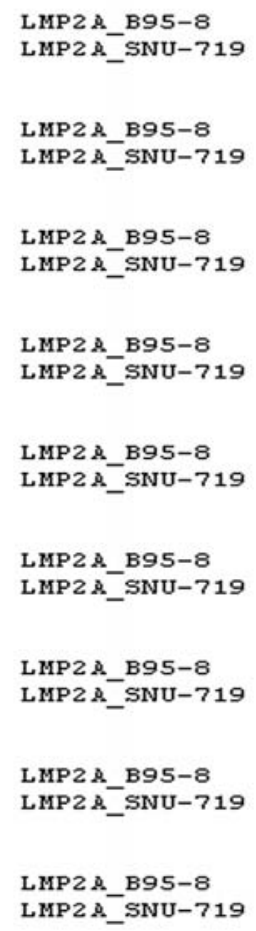

Figure 2. Sequence alignment of SNU-719 LMP2A with B95-8 LMP2A. Open boxes indicate changed amino acids of SNU-719 LMP2A compared to the B95-8 LMP2A sequences. There are eight amino acid differences. The asterisks $(*)$ indicate tyrosine residues. Grey boxes indicate 12 transmembrane domains.

Cell growth in reduced serum medium. Cells $\left(7 \times 10^{3} /\right.$ well) in a medium containing $10 \%$ FBS were seeded in a 12 -well plate. After one day, the cells were washed once with a serum-free medium and supplied with a fresh medium containing $1 \%$ FBS The cells were then counted once every other day for 5 days.

\section{Results}

Cloning and sequence analysis of LMP2A gene from SNU-719.

A 1,511 bp long LMP2A transcript was RT-PCR amplified from the SNU-719 cell line (Fig. 1A). The RT-PCR product was cloned into the retroviral vector pMIG (Fig. 1B). Escherichia coli transformed with the LMP2A construct, was used to purify DNA for the sequencing of the construct. The resulting LMP2A sequence comparison between SNU-719 and B95-8 prototype strain is shown in Fig. 2. The LMP2A of SNU-719 showed six changes at amino acids 23, 38, 63, 64,79 and 82 in the N-terminus and two changes ( $\mathrm{S}$ to $\mathrm{T}$ ) at amino acids 348 and 444 in the inter-membrane domains compared to the LMP2A of B95-8. 
A

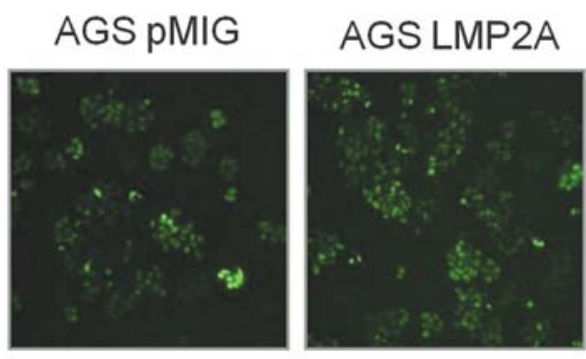

B

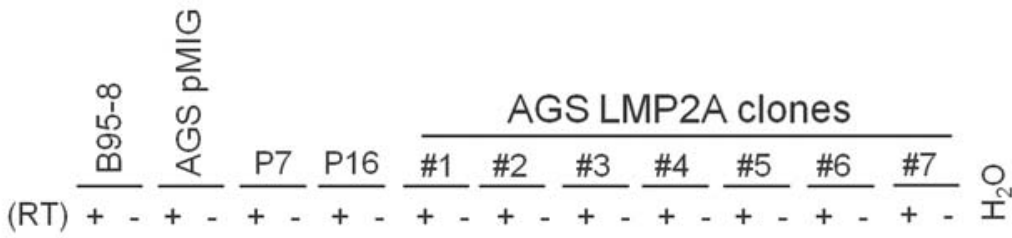

\begin{tabular}{|c|c|c|c|c|c|c|c|c|c|c|}
\hline LMP2A & Ë- & - & - & - & - & - & - & - & - & - \\
\hline GAPDH & -- & - & - & - & - & - & - & - & - & - \\
\hline
\end{tabular}

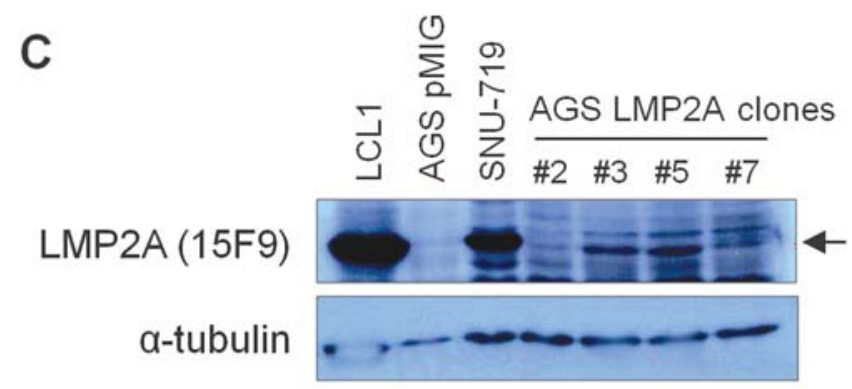

Figure 3. Detection of LMP2A gene expression in the AGS cell clones transduced with the LMP2A construct. Permanent cell clones were obtained by serial dilution. Detection of GFP expression in the AGS cell clones transduced with LMP2A gene after FACS sorting (A). To analyze the expression of LMP2A in each cell clone, total RNA was extracted and then RT-PCR was performed. For comparison, RT-PCR was also performed for GAPDH. P7 and P16 indicate passages 7 and 16 of subculture (B). LMP2A protein was detected by Western blot assay with anti-15F9 antibody. The arrow indicates the LMP2A band (C).

LMP2A gene transduction into AGS cells and isolation of single cell clones. The LMP2A gene cloned in the pMIG retroviral vector was transduced into the EBV-negative gastric cancer cell line, AGS. After GFP expression was confirmed (Fig. 3A), the transduced cells were maintained for one week and the GFP expressing cells were collected by FACS. The mRNA expression of LMP2A gene in the sorted cells was verified by RT-PCR after four passages (data not shown). Cell clones expressing LMP2A were then isolated by serial dilution. Seven cell clones were obtained and the expression of LMP2A mRNA was detected by RT-PCR (Fig. 3B). To confirm the expression of LMP2A protein in the cell clones, Western blot assay was carried out. As shown in Fig. 3C, four (\#2, \#3, \#5, and \#7 clones) out of seven LMP2A-transduced cell clones expressed LMP2A protein at a detectable level. However, the expression level of LMP2A in the cell clones was lower than the levels observed in SNU-719 and LCL1 used as positive controls.

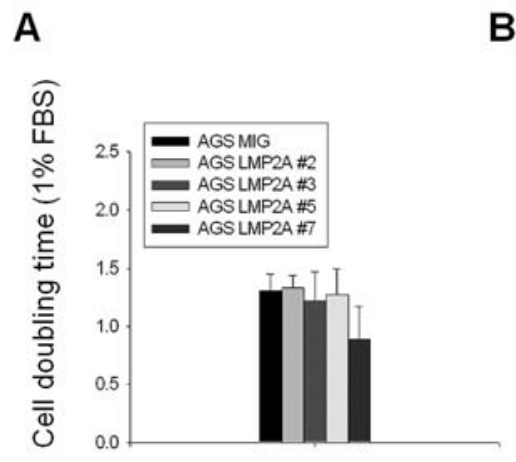

B
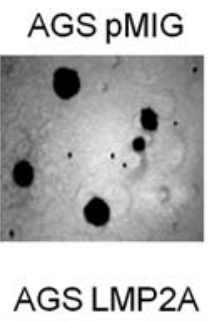

Figure 4. Effect of LMP2A gene expression on cell growth. LMP2A clones were cultured in medium containing $1 \%$ FBS and counted after 5 days. The data represent mean \pm SEM of three independent experiments $(n=3)(A)$. To investigate an anchorage-independent growth ability, cells were seeded in soft agar and then cultured for 3 weeks. Colonies were stained with $0.005 \%$ crystal violet for visualization and then washed with de-ionized water (B). 
Effect of LMP2A on the cell growth. The effect of LMP2A gene on the cell growth was analyzed by culturing the cells in medium containing low serum (1\% FBS). LMP2A expressing cell clones had little or no effect on cell growth when compared with the AGS pMIG (Fig. 4A). The growth rate of these cell clones was not different from that of AGS pMIG cells in $10 \%$ FBS-containing medium, either (data not shown). The colony formation efficiency (CFE) of the cell clones expressing LMP2A gene was evaluated in soft agar in duplicate experiments (Fig. 4B). EBV-negative AGS cells that were

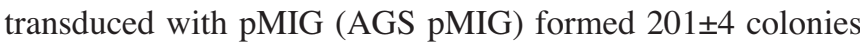
(mean \pm standard deviation, $\mathrm{CFE}=0.13 \%$ ). EBV-positive AGS cells (AGS-EBV) formed $193 \pm 2$ colonies $(\mathrm{CFE}=0.13 \%)$. The LMP2A expressing cell clone formed $218 \pm 2$ colonies $(\mathrm{CFE}=0.15 \%)$, which was comparable with AGS-EBV and AGS pMIG cells.

\section{Discussion}

EBV infection is associated with $\sim 10 \%$ of the GC worldwide. However, the causal relationship between EBV and EBVaGC remains unclear. EBVaGC exerts a modified latency I and expresses only a limited set of latent EBV proteins such as EBNA-1 and LMP2A. LMP2A is routinely detected in HD, $\mathrm{BL}, \mathrm{NPC}$ and GC, suggesting that it may contribute to EBVassociated tumor formation and/or maintenance. However, the exact role(s) of LMP2A in EBV-associated tumors is contentious. To better understand the role of LMP2A in EBVaGC, we constructed LMP2A-expressing cell clones by transducing LMP2A gene into an EBV-negative gastric carcinoma cell line AGS.

SNU-719 LMP2A showed six sequence variations at the LMP2A N-terminal domain. Among eight tyrosine residues within the N-terminus of LMP2A, Y23 and Y64 were changed (Y to D) while Y31, Y60, Y74, Y85, Y101, and Y112 were not altered. Y74, Y85 and Y112 have been demonstrated to be essential for LMP2A-mediated blockade of protein phosphorylation, calcium mobilization, and induction of BZLF1 expression after BCR activation, while the other tyrosine residues did not affect LMP2A function in vitro (30-32). Thus, $\mathrm{Y}$ to D changes at Y23 and Y64 are expected to have little effect on the function of LMP2A in SNU-719. SNU-719 LMP2A showed additional amino acid substitutions at amino acids 348 and 444 of the inter-membrane domains. The $S \rightarrow T$ substitution at the amino acid 348 of LMP2A which is located in the CTL-epitope region, is a frequent event in EBVaGC cases (33). Such alterations in CTL-epitopes of LMP2A may have helped to avoid immune attack of the EBV-associated gastric carcinoma from which SNU-719 was derived.

After transduction with the retrovirus co-expressing EGFP and LMP2A, cell clones continued to express EGFP for at least 20 passages (120-140 days after gene transduction). This suggests the integration of the LMP2A gene as well as the EGFP gene into the cellular chromosome. However, the intensity of GFP expression was variable in the established cell clones, implying different integration sites or extents of integration.

In our experiments, LMP2A expression did not contribute a growth advantage to AGS cells in low serum medium and LMP2A did not enhance anchorage-independent growth of
AGS cells. There are several plausible reasons for this. First, LMP2A does not have any effect on cell growth, transformation, and tumorigenesis of gastric epithelial cells. Second, the oncogenic effect of the LMP2A gene may have been masked by the basal cell activity because AGS itself is a GC cell line. Indeed, AGS cells showed comparable growth ability in soft agar not only with AGS LMP2A expressing cell clones but also with EBV-positive AGS cells. Third, there might not be enough LMP2A expressed to function properly. We observed that LMP2A was expressed only at low level in all the AGS LMP2A clones compared to the EBV infected cell lines. Compared to the episomal EBV genomes present at up to 50 copies within a cell $(34,35)$, the retroviral vector we used is expected to be integrated to the chromosomal DNA at one or a few different sites. Gene silencing may also have contributed to low LMP2A expression in AGS cell clones, as retroviral vectors often suffer from gene silencing after they are integrated to the chromosomal DNA (36).

In addition, expression of LMP2A gene by itself may not sufficient to affect cell growth leading to oncogenesis, rather they might co-operate to cause tumors with other EBV genes. Related to this point, EBV has been recently shown to express over 20 viral microRNAs (miRNA) $(37,38)$. miRNAs have variable effects and they may cooperate with the latent EBV genes to drive tumorigenesis. In order to identify possible contribution of the latent EBV genes to the development of epithelial malignancies, further analysis of cell lines coexpressing two or more EBV genes would be required.

In conclusion, we established LMP2A gene-expressing cell clones and found that LMP2A by itself does not enhance cell growth any further in an EBV-negative GC cell line.

\section{Acknowledgements}

This study was supported by a grant from the National R\&D Program for Cancer Control, Ministry of Health and Welfare, Republic of Korea (0620320) and by Korea Research Foundation Grant (KRF-2004-015-E00190). J.S. Seo was supported by the Seoul Science Fellowship.

\section{References}

1. Rickinson A: Epstein-Barr virus. Virus Res 82: 109-113, 2002.

2. Takada K, Shimizu N, Tanabe-Tochikura A and Kuroiwa Y: Pathogenic role of Epstein-Barr virus in human cancer. Intervirology 38: 214-220, 1995.

3. Young LS and Murray PG: Epstein-Barr virus and oncogenesis: from latent genes to tumours. Oncogene 22: 5108-5121, 2003.

4. Uozaki H and Fukayama M: Epstein-Barr virus and gastric carcinoma - viral carcinogenesis through epigenetic mechanisms. Int J Clin Exp Pathol 1: 198-216, 2008.

5. Imai S, Koizumi S, Sugiura M, et al: Gastric carcinoma: monoclonal epithelial malignant cells expressing Epstein-Barr virus latent infection protein. Proc Natl Acad Sci USA 91: 9131-9135, 1994.

6. Fukayama M, Hayashi Y, Iwasaki Y, et al: Epstein-Barr virusassociated gastric carcinoma and Epstein-Barr virus infection of the stomach. Lab Invest 71: 73-81, 1994.

7. zur Hausen A, van Grieken NC, Meijer GA, et al: Distinct chromosomal aberrations in Epstein-Barr virus-carrying gastric carcinomas tested by comparative genomic hybridization. Gastroenterology 121: 612-618, 2001.

8. Nishikawa J, Imai S, Oda T, Kojima T, Okita K and Takada K: Epstein-Barr virus promotes epithelial cell growth in the absence of EBNA2 and LMP1 expression. J Virol 73: 1286-1292, 1999. 
9. zur Hausen A, Brink AA, Craanen ME, Middeldorp JM Meijer CJ and van den Brule AJ: Unique transcription pattern of Epstein-Barr virus (EBV) in EBV-carrying gastric adenocarcinomas: expression of the transforming BARF1 gene. Cancer Res 60: 2745-2748, 2000.

10. Sugiura M, Imai S, Tokunaga M, et al: Transcriptional analysis of Epstein-Barr virus gene expression in EBV-positive gastric carcinoma: unique viral latency in the tumour cells. Br J Cancer 74: 625-631, 1996.

11. Miller CL, Lee JH, Kieff E, Burkhardt AL, Bolen JB and Longnecker R: Epstein-Barr virus protein LMP2A regulates reactivation from latency by negatively regulating tyrosine kinases involved in sIg-mediated signal transduction. Infect Agents Dis 3: 128-136, 1994

12. Portis $T$ and Longnecker R: Epstein-Barr virus (EBV) LMP2A mediates B-lymphocyte survival through constitutive activation of the Ras/PI3K/Akt pathway. Oncogene 23: 8619-8628, 2004.

13. Anderson LJ and Longnecker R: EBV LMP2A provides a surrogate pre-B cell receptor signal through constitutive activation of the ERK/MAPK pathway. J Gen Virol 89: 1563-1568, 2008.

14. Swart R, Ruf IK, Sample J and Longnecker R: Latent membrane protein 2A-mediated effects on the phosphatidylinositol 3-kinase/ Akt pathway. J Virol 74: 10838-10845, 2000.

15. Fukuda $\mathrm{M}$ and Longnecker R: Latent membrane protein $2 \mathrm{~A}$ inhibits transforming growth factor-beta 1-induced apoptosis through the phosphatidylinositol 3-kinase/Akt pathway. J Virol 78: 1697-1705, 2004.

16. Kim OJ and Yates JL: Mutants of Epstein-Barr virus with a selective marker disrupting the TP gene transform B cells and replicate normally in culture. J Virol 67: 7634-7640, 1993.

17. Longnecker R, Miller CL, Miao XQ, Marchini A and Kieff E: The only domain which distinguishes Epstein-Barr virus latent membrane protein 2A (LMP2A) from LMP2B is dispensable for lymphocyte infection and growth transformation in vitro; LMP2A is therefore nonessential. J Virol 66: 6461-6469, 1992.

18. Mancao C and Hammerschmidt W: Epstein-Barr virus latent membrane protein $2 \mathrm{~A}$ is a $\mathrm{B}$-cell receptor mimic and essential for B-cell survival. Blood 110: 3715-3721, 2007.

19. Scholle F, Bendt KM and Raab-Traub N: Epstein-Barr virus LMP2A transforms epithelial cells, inhibits cell differentiation, and activates Akt. J Virol 74: 10681-10689, 2000.

20. Chen SY, Lu J, Shih YC and Tsai CH: Epstein-Barr virus latent membrane protein $2 \mathrm{~A}$ regulates c-Jun protein through extracellular signal-regulated kinase. J Virol 76: 9556-9561, 2002.

21. Pegtel DM, Subramanian A, Sheen TS, Tsai CH, Golub TR and Thorley-Lawson DA: Epstein-Barr-virus-encoded LMP2A induces primary epithelial cell migration and invasion: possible role in nasopharyngeal carcinoma metastasis. J Virol 79: 15430-15442, 2005.

22. Fukuda M and Longnecker R: Epstein-Barr virus latent membrane protein 2A mediates transformation through constitutive activation of the Ras/PI3-K/Akt pathway. J Virol 81: 9299-9306, 2007.
23. Longan L and Longnecker R: Epstein-Barr virus latent membrane protein $2 \mathrm{~A}$ has no growth-altering effects when expressed in differentiating epithelia. J Gen Virol 81: 2245-2252, 2000.

24. Oh ST, Seo JS, Moon UY, et al: A naturally derived gastric cancer cell line shows latency I Epstein-Barr virus infection closely resembling EBV-associated gastric cancer. Virology 320: 330-336, 2004

25. Park JG, Yang HK, Kim WH, et al: Establishment and characterization of human gastric carcinoma cell lines. Int J Cancer 70: 443-449, 1997.

26. Barranco SC, Townsend CM Jr, Casartelli C, et al: Establishment and characterization of an in vitro model system for human adenocarcinoma of the stomach. Cancer Res 43: 1703-1709, 1983.

27. Yoshiyama H, Imai S, Shimizu N and Takada K: Epstein-Barr virus infection of human gastric carcinoma cells: implication of the existence of a new virus receptor different from CD21. J Virol 71: 5688-5691, 1997.

28. Oh IH and Eaves CJ: Overexpression of a dominant negative form of STAT3 selectively impairs hematopoietic stem cell activity. Oncogene 21: 4778-4787, 2002.

29. Wigler M, Pellicer A, Silverstein S and Axel R: Biochemical transfer of single-copy eucaryotic genes using total cellular DNA as donor. Cell 14: 725-731, 1978 .

30. Swart R, Fruehling S and Longnecker R: Tyrosines 60, 64, and 101 of Epstein-Barr virus LMP2A are not essential for blocking B cell signal transduction. Virology 263: 485-495, 1999.

31. Fruehling S, Swart R, Dolwick KM, Kremmer E and Longnecker R: Tyrosine 112 of latent membrane protein $2 \mathrm{~A}$ is essential for protein tyrosine kinase loading and regulation of Epstein-Barr virus latency. J Virol 72: 7796-7806, 1998.

32. Fruehling $\mathrm{S}$ and Longnecker R: The immunoreceptor tyrosinebased activation motif of Epstein-Barr virus LMP2A is essential for blocking BCR-mediated signal transduction. Virology 235: 241-251, 1997.

33. Tanaka M, Kawaguchi Y, Yokofujita J, Takagi M, Eishi Y and Hirai K: Sequence variations of Epstein-Barr virus LMP2A gene in gastric carcinoma in Japan. Virus Genes 19: 103-111, 1999.

34. zur Hausen H, Schulte-Holthausen H, Klein G, et al: EBV DNA in biopsies of Burkitt tumours and anaplastic carcinomas of the nasopharynx. Nature 228: 1056-1058, 1970.

35. zur Hansen H, Diehl V, Wolf H, Schulte-Holthausen H and Schneider U: Occurrence of Epstein-Barr virus genomes in human lymphoblastoid cell lines. Nat New Biol 237: 189-190, 1972.

36. McTaggart S and Al-Rubeai M: Retroviral vectors for human gene delivery. Biotechnol Adv 20: 1-31, 2002.

37. Kim do N, Chae HS, Oh ST, et al: Expression of viral microRNAs in Epstein-Barr virus-associated gastric carcinoma. J Virol 81: 1033-1036, 2007

38. Pfeffer S, Zavolan M, Grasser FA, et al: Identification of virusencoded microRNAs. Science 304: 734-736, 2004. 